\title{
Die Lausitz als Modellregion einer nachhaltigen Transformation
}

\author{
Eine qualitative Inhaltsanalyse auf Basis der 17 Sustainable \\ Development Goals zur Integration von sozialer, ökologischer und \\ ökonomischer Entwicklung
}

\author{
Alexandra Retkowski
}

Eingegangen: 1. Mai 2021 / Angenommen: 15. Juni 2021 / Online publiziert: 27. September 2021 (C) Der/die Autor(en) 2021

Zusammenfassung Seit dem im Jahre 2019 beschlossenen Kohleausstieg ist die Lausitz als eine der drei bundesrepublikanischen Kohlereviere ins Bewusstsein einer breiteren Öffentlichkeit gerückt. Es handelt sich um eine Region, die sich nach dem dreißigjährigen Transformationsprozess im Zuge des Einheitsprozesses dadurch erneut in einem tiefgreifenden Strukturwandel befindet. Der Beitrag geht auf Basis einer qualitativen Inhaltsanalyse von Positionspapieren verschiedener Lausitzer Akteur*innengruppen aus Politik, Wirtschaft und Zivilgesellschaft der Frage nach, wie sich in diesen Stellungnahmen Forderungen und Zielvorstellungen eines sozialökologischen Wandlungsprozesses abbilden. Als Kategoriensystem wird auf die 17 Sustainable Development Goals (SDGs) der von der Bundesrepublik unterzeichneten AGENDA 2030 der United Nations (UN) zurückgegriffen, die mit den fünf Kernbotschaften People, Prosperity, Planet, Partnership und Peace ein Programm zur ökonomischen, ökologischen und sozialen Nachhaltigkeit im Sinne eines integrativen Nachhaltigkeitskonzepts entwickelt hat. Das empirische Vorgehen verfolgt ein doppeltes Ziel: Es werden die Gemeinsamkeiten zwischen den Perspektiven der Lausitzer Positionspapiere und den 17 SDGs herausgearbeitet, sodass auf dieser Basis das Profilbild eines möglichen Lausitzer Transformationsprozesses entlang der AGENDA 2030 entsteht. Zum anderen wird die Bedeutung der Sozialen Arbeit innerhalb dieses Profilbilds ausgelotet und im Anschluss diskutiert, welche Potenziale die Nachhaltigkeitsorientierung der 17 SDGs für die Soziale Arbeit hat.

Schlüsselwörter Sustainable Development Goals · Qualitative Inhaltsanalyse · Lausitzer Strukturwandel · Nachhaltigkeit · Soziale Arbeit

Prof. Dr. Alexandra Retkowski $(\bowtie)$

Brandenburgische Technische Universität, Cottbus-Senftenberg Lipezker Straße 47, 03084 Cottbus, Deutschland

E-Mail: alexandra.retkowski@b-tu.de 


\section{Lusatia as a model region of sustainable transition}

A qualitative content analysis based on the 17 sustainable development goals on integration of social, ecological and economic development

Abstract The decision to phase out coal in 2019 has brought Lusatia, one of the three affected coal regions in the Federal Republic of Germany, to the attention of a broader public. After 30 years of transformation as part of the unification process, the region is once again undergoing a profound structural change. On the basis of a qualitative content analysis of the position papers of various Lusatian groups of players from politics, business and civil society, the article investigates the question of how demands and objectives of a socioecological process of change are reflected in these statements. The 17 sustainable goals (sustainable development goals or 17 SDGs) of the AGENDA 2030 of the United Nations (UN) signed by the Federal Republic of Germany serve as the system of categories. The five core messages people, prosperity, planet, partnership and peace outline a program for economic, ecological and social sustainability in the sense of an integrative sustainability concept. The empirical approach pursues a double goal: On the one hand, the connecting lines between the perspectives of the Lusatian position papers and the 17 SDGs are identified, so that the profile picture of a possible Lusatian transformation process based on the AGENDA 2030 is created. On the other hand, the importance of social work is explored within the framework of this Lusatian transformation process. It is discussed what potential the socioecological development program of the 17 SDGs has for social work.

Keywords Sustainable development goals (SDG) - Qualitative content analysis · Lusatian structural transformation - Sustainability and social work

\section{Einleitung}

In seinem „Brief aus Berlin“ machte C. Wolfgang Müller bereits im Jahr 2016 auf den Lausitzer Strukturwandel aufmerksam: „Nach der Wende verlor sie zunächst den (politischen) Konkurrenzkampf mit der Steinkohle in NRW, danach die Auseinandersetzung mit erneuerbaren Energien“, sodass „der Verlust der Kohle für alteingesessene Lausitzer nicht nur den Verlust von Arbeit, sondern auch von Heimat und Identität" bedeute (Müller 2016, S. 60). Damit ist bereits der enorme ökonomische, ökologische und soziokulturelle Wandlungsprozess angedeutet, in dem sich die Lausitz - eine Region in Teilen von Brandenburg, Sachsen und Polen gelegen - als zweitgrößtes bundesdeutsches Braunkohlerevier mit dem Prozess des Ausstiegs aus der Braunkohleverstromung befindet. C. W. Müller beschrieb den Prozess als doppelte Verlustgeschichte: Verlust als sozialen Abschied von traditionsreichen Wissens- und Kulturbeständen wie auch als ökonomische Abkehr von einem regionalen industriellen Wirtschaftsmodell, bei dem die Bruttowertschöpfung je Erwerbstätigem über dem ostdeutschen Durchschnitt lag - verbunden mit entsprechenden Gehältern und industriewirtschaftlich geprägten Arbeitsplatzmodellen (Seibert et al. 2018, S. 52). Mit dieser regionalen Krisenbeschreibung ist die globale 
Krise der Klimaerhitzung noch gar nicht in den Fokus gerückt, die es notwendig macht, den $\mathrm{CO}_{2}$-Ausstoß so weit zu reduzieren, dass die Erderwärmung deutlich unter zwei Grad gegenüber dem vorindustriellen Niveau verbleibt. Die Lausitz wird in diesem Zusammenhang oftmals als „Modellregion“ betrachtet, wie der damit notwendige energiewirtschaftliche Strukturwandel auch sozial gelingt, denn es sind oftmals die Reformen zur Bewältigung der Klimakrise, die vor Ort als Krise und Generator sozialer Ungleichheiten erlebt werden. Somit werden durch den Lausitzer Transformationsprozess ,die Überschneidungs- und Verdrängungsbeziehungen zwischen sozialer und ökologischer Frage“ (Beck 1992, S. 198) deutlich offenbar.

Blickt man in den diesbezüglichen wissenschaftlichen Diskurs, so zeigt sich, dass zwar „Klima“ sukzessive nicht mehr nur ein Gegenstand naturwissenschaftlicher Forschung ist, sondern auch in den Sozial- und Humanwissenschaften aufgegriffen wird und die Frage der Integration des Nachhaltigkeitsthemas auch in den Blick der Sozialen Arbeit gerät (Böhnisch 2020; Liedholz 2021). Ebenfalls existiert in der Sozialen Arbeit eine lange Tradition der Auseinandersetzung mit krisenhaften, gesellschaftlichen Transformationsprozessen - gerade auch mit Blick auf Ostdeutschland und ihren Auswirkungen auf die Lebenslagen von Adressat*innen, der Analyse und Reflexion von Auswirkungen auf die Angebots- und professionellen Handlungsformen der Sozialen Arbeit sowie der sich daraus ergebenden (sozial)politischen Mandatierung (Alheit et al. 2004; Bock 2000; Bütow et al. 2014; Bütow et al. 2006; Olk und Gensicke 2014). Der Beitrag setzt in diesem Kontext beim Lausitzer Strukturwandel an, indem mit Hilfe einer qualitativen Inhaltsanalyse von Positionspapieren analysiert wird, wie von lokalen Stakeholdern wirtschaftliche, soziale und ökologische Nachhaltigkeit im Sinne der 17 Nachhaltigkeitsziele (SDGs) der UN entworfen wird. Für dieses Ziel wird zunächst die Programmatik der 17 SDGs sowie der wissenschaftliche Diskurs über sie vorgestellt (2). Dem schließt sich die Darstellung des methodischen Vorgehens der qualitativen Inhaltsanalyse an, wobei die 17 Nachhaltigkeitsziele als Modell für die Kategorienbildung zu Grunde gelegt werden (3). Im vierten Kapitel werden die Ergebnisse der Inhaltsanalyse aufgeführt (4). Dem folgt eine Reflexion zum Strukturwandel der Lausitz im Lichte der 17 SGGs und ihrer Thematisierung in den analysierten Positionspapieren (5). Abgeschlossen wird der Beitrag im sechsten Kapitel mit einem Ausblick zu den Potenzialen der Sozialen Arbeit innerhalb sozial-ökologischer Transformationsprozesse im Sinne der Sustainable Development Goals der UN (6).

\section{Sustainable Development Goals - die Integration von sozialen, ökonomischen und ökologischen Perspektiven}

Im Jahre 2015 haben bei der Generalversammlung der United Nations 193 Länder die AGENDA 2030 mit ihren 17 Nachhaltigkeitszielen (Sustainable Development Goals) und ihren 167 Unterzielen als Nachfolge und Präzisierung der Millennium Development Goals unterzeichnet. Die Agenda ist ein gemeinsam verabschiedeter Fahrplan für die internationale, europäische und bundesdeutsche Umsetzung eines integrierten ökologischen, sozialen und ökonomischen Nachhaltigkeitskonzepts, welches sich an Politik, Zivilgesellschaft, Wirtschaft und Wissenschaft mit dem 
Ziel richtet, weltweit ein menschenwürdiges Leben zu ermöglichen und dabei die natürlichen Lebensgrundlagen dauerhaft zu bewahren. (Bundesregierung 2021). In Verbindung mit dem Pariser Klima-Abkommen geht es um den weltweiten Wandel hin zu nachhaltigen und emissionsarmen Lebens- und Wirtschaftsweisen (BMZ 2021). Der bundesdeutsche Kohleausstieg und die schrittweise Abschaltung der Kohlekraftwerke in der Lausitz bis spätestens 2038 stehen in diesem Zusammenhang.

Mit den SDGs ist ein Dreisäulenmodell der Nachhaltigkeit in Bezug auf den Umgang mit Ressourcen verbunden, wonach das Kriterium der „Effizienz“ im wirtschaftlichen Bereich die ergiebigere Nutzung von Ressourcen durch technische und logistische Innovationen beschreibt, „Konsistenz“ eine Orientierung im ökologischen Bereich definiert, die Stoff- und Energieströme so ausrichtet, dass die Leistungen der Ökosysteme genutzt werden, ohne sie zu zerstören, und „Suffizienz“ im sozialen Bereich auf einen „Wandel der Einstellungen, Naturverhältnisse, der Konsum- und Gesundheits-, Gerechtigkeits- und Verhaltensmuster“ im Dienste der Verringerung des Ressourcenverbrauchs abzielt (Opielka 2016; Verch 2020).

Blickt man in die wissenschaftliche Auseinandersetzung um die AGENDA 2030 mit ihren 17 SDGs, so zeigt sich, dass die Programmatik aus unterschiedlichen Perspektiven kritisch diskutiert wird. Zunächst gibt es Kritik an der Systematik des Programms. Es wird also die Frage aufgeworfen, ,wie die Funktionsweise des Systems von Nachhaltigkeitsnormen insgesamt zu denken ist" und wie dabei mit Zielkonflikten zwischen den einzelnen Normbereichen umgegangen werden kann (Dusseldorp 2017, S. 232). Zweitens ist der Implementierungsprozess Gegenstand der Kritik. So wird die hohe und dadurch verwirrende Anzahl der Einzel- und Unterziele problematisiert, die die Programmatik als „Christmas tree“ (Langford 2016, S. 168) erscheinen lasse. Des Weiteren sei die Implementierung technologisch und managementorientiert ausgerichtet (Bruns 2019), und es gäbe nur geringe Überprüfungs- und Evaluierungsprozesse (Beisheim 2014). Daher bestehe die Gefahr einer ,governance by indicators“ und der Orientierung einer Logik an ,big data“ ohne gesellschaftlich getragene Partizipationsprozesse (Fukuda-Parr und McNeill 2019). Inhaltlich besteht die Kritik, dass die mit den 17 SDGs verbundene weiter vorangetriebene Globalisierung Ungleichheiten nicht verringere, sondern verstärke (Schmieg 2017). Während zwar soziale und ökologische Aspekte betont würden, würde das wachstumsorientierte Wirtschaftsmodell ebenso wenig in Frage gestellt wie die damit verbundenen Machtverhältnisse (Stevens und Kanie 2016). Die SDGs seien somit ein ,great gift to business“ (Pedersen 2018). Schließlich wird auch die ökologische Effektivität der UN-Programmatik in Frage gestellt. Es wird problematisiert, dass selbst im Fall einer Erreichung aller Ziele der Agenda 2030, dies weder für eine Reduktion der Ressourcennutzung bis zu einem Level planetarischer Grenzen noch für die nachhaltige Veränderung gesellschaftlicher Strukturen ausreichend wäre (Eisenmenger et al. 2020).

Trotz dieser erheblichen Einwände wird in diesem Beitrag positiv auf die 17 SDGs und die AGENDA 2030 Bezug genommen und als deduktives Kategoriensystem für die qualitative Inhaltsanalyse zu Grunde gelegt. Dies geschieht aus drei Gründen. Erstens wird der Auffassung gefolgt, dass die 17 SDGs das ambitionierteste Projekt eines universellen Entwicklungsmodells sind, welches das Ziel einer sicheren, gerechten und inklusiven Welt für alle Menschen in einer gemeinsamen Verantwor- 
tung für Nachhaltigkeit verwirklichen möchte (Filho 2019; Herlyn und Lévy-Tödther 2020). Darüber hinaus ist für den Prozess der Internationalisierung der Sozialen Arbeit die Auseinandersetzung mit internationalen Dokumenten und Strategiepapieren von besonderer Bedeutung (Lutz et al. 2018). Drittens waren bereits die UN-Kinderrechtskonvention aus dem Jahre 1992 und die UN-Behindertenrechtskonvention aus dem Jahre 2008 für das bundesdeutsche Sozial- und Bildungswesen von paradigmatischer Bedeutung. Ebenso kann das transformative Potenzial der Sozialen Arbeit in der Agenda 2030 als für Regierungen verbindlichem UN-Dokument eine großes Entfaltungspotenzial bieten (Spitzer 2019). Aktuell werden bereits für den Bereich der Kinder- und Jugendhilfe beispielsweise „ökologische Kinderrechte“ und die Relevanz der 17 SDGs diskutiert (AGJ 2020).

\section{Methodisches Vorgehen}

Die Analyse der Positionspapiere zum Lausitzer Strukturwandel erfolgt in Anlehnung an die qualitative Inhaltsanalyse, die Philip Mayring als ,qualitativ orientierte kategoriengeleitete Textanalyse“ beschreibt (Mayring 2020, S. 500). Als Kategoriensystem wurden die 17 Nachhaltigkeitskriterien der United Nations zu Grunde gelegt. Die inhaltlich sehr breit angelegten 17 Ziele mit ihren 169 Unterzielen bzw. Zielindikatoren sind in fünf Kernbotschaften (Peace, Partnerhip, People, Planet, Prosperitiy) unterteilt, welche die Ziele entlang thematischer Schwerpunktsetzungen differenzieren und ,die Zusammenhänge zwischen den Zielen verdeutlichen“ sollen (BMZ 2017 S. 7). Was zu Beginn als plausible Strukturierung erschien, erwies sich im Fortgang der Untersuchung als problematisch. So zeigte die Recherche zum Kategoriensystem erstens, dass die Definition der Kernbotschaften extrem unterschiedlich vorgenommen wird. Von Uwe Schneidewind werden die Kernbotschaften, die sogenannten ,5 Ps“, wie folgt charakterisiert:

(1) People, d.h. die Sicherstellung der Grundbedingungen für ein menschenwürdiges Leben, wie die Verhinderung von Armut und Hunger, der Zugang zu Wasser und Strom, aber auch zu Bildung und Gesundheit. (2) Prosperity, d.h. der Anspruch auf einen über diese Grundbedürfnisse hinausgehenden Wohlstand für alle Menschen. (3) Diese menschlichen Entwicklungsziele werden mit dem Erhalt der ökologischen Grundlagen der Erde (Planet) verknüpft: Klimaschutz, Schutz der Meere, Schutz der Landökosysteme. Zudem sagen die SDGs etwas darüber, wie die obengenannten Ziele global umzusetzen sind. Hier sind (4) die Formen des friedlichen Umgangs (Peace) der Nationen miteinander sowie (5) das Prinzip der globalen Partnerschaft (Partnership) zentrale Anliegen“ (Schneidewind 2018, S. 113).

Greift man beispielsweise die Kernbotschaft Prosperity heraus, so zeigt sich, dass die UN die Kernbotschaft als ,We are determined to ensure that all human beings can enjoy prosperous and fulfilling lives and that economic, social and technological progress occurs in harmony with nature" definiert ${ }^{1}$. Vom bundesrepublikanischen Bundesministerium für wirtschaftliche Zusammenarbeit und Entwicklung

\footnotetext{
1 Online unter https://sdgs.un.org/2030agenda [Letzter Zugriff am 15. Juni 2021].
} 
(BMZ) wird diese als „Globalisierung gerecht gestalten“ bezeichnet und wie folgt beschrieben:

„Die Wirtschaft ist Wohlstandstreiber und Entwicklungsmotor. Das BMZ trägt dazu bei, allen Menschen weltweit die Chance zu bieten, am wirtschaftlichen, sozialen und technischen Fortschritt teilzuhaben. Nachhaltiges Wirtschaftswachstum muss alle Menschen erreichen und ihnen eine menschenwürdige Arbeit und Beschäftigung bieten. Die Schere zwischen Arm und Reich darf dabei nicht noch weiter auseinandergehen." 2

Allein in den hier aufgeführten drei Definitionen seitens der UN, von Scheidewind und vom BMZ zeigt sich, dass zwar soziale Gerechtigkeit in globaler Perspektive das verbindende Element der definierten Entwicklungsziele ist, diese aber unterschiedlich konnotiert sind und weder Schneidewind noch das BMZ den Passus der UN zur Definition von Prosperity als einer Entwicklung ,in harmony with nature“ in ihre Definitionen aufgenommen haben. Mit dieser Unklarheit und Uneindeutigkeit der Definition der Kernbotschaften geht die Gefahr einer geringeren Verknüpfung und Integration der einzelnen Kernbereiche einher.

Zweitens zeigte sich, dass hinsichtlich der Zuordnung der 17 SDGs zu den fünf Kernbotschaften sehr viele unterschiedliche Verwendungsweisen existieren. Je nach Zuordnung und Gruppierung verweist dies sowohl auf sehr unterschiedliche Hintergrundkonstruktionen und Verständnisweisen der Kernbotschaften wie auch auf konkrete Umsetzungsziele der einzelnen SDGs.

Drittens existieren Systematiken, die nicht auf die fünf Kernbotschaften Bezug nehmen. Die Zuordnung, die sich beispielsweise auf Wikipedia befindet, weist nur drei Unterteilungen auf, bei der die Dimension des Wirtschaftlichen ganz fehlt: „Spiritual“, „Ecological“ und „People“. ${ }^{3}$ Die in der internationalen Literatur zu den 17 SDGs vertretene Auffassung, die Zuordnung der Ziele und Indikatoren sei nicht zielführend und unsystematisch (Elder und Olsen 2019), kann hier also bestätigt werden.

In der hier vorgenommenen qualitativen Inhaltsanalyse wurde als deduktives Kategoriensystem auf die Zuordnung der UN zurückgegriffen, da anzunehmen ist, dass dies die erste festgeschriebene und veröffentlichte Version ist. Dort werden die 17 SDGs entsprechend ihrer chronologischen Nummerierung den fünf Kernbotschaften zugeordnet (Tab. 1).

Die 17 SDGs gemäß der UN-Systematik wurden also als analytische Kategorien an das Datenmaterial herangetragen und zur inhaltlichen Strukturbildung genommen. Das Ziel dieser deduktiven bzw. A-priori-Kategorienanwendung (Kuckartz 2018, S. 64) bestand darin herauszufinden, ob, in welcher Ausprägung und wie sich die SDGs in den Positionspapieren zum Lausitzer Strukturwandel wiederfinden lassen. Mit anderen Worten stellten die fünf Kerndimensionen den Kodierleitfaden dar und wurden als „Suchraster“ (Kuckartz 2018, S. 96) genommen. Der Kodierprozess wurde mittels der Intra- und Interkoderreliabilität überprüft, und bei

\footnotetext{
2 Online abrufbar unter: https://www.bmz.de/de/agenda-2030 [Letzter Zugriff am 15. Juni 2021].

3 Online abrufbar unter: https://en.wikipedia.org/wiki/Sustainable_Development_Goals\#/media/File: SDG-pyramid.jpg. Als „Spiritual“ werden dabei die Dimensionen Peace und Partnership gefasst.
} 
Tab. 1 Fünf Kernbotschaften der SDGs (eigene Darstellung)

\begin{tabular}{|c|c|c|c|c|}
\hline People & Prosperity & Planet & Peace & Partnership \\
\hline 1. Keine Armut & $\begin{array}{l}\text { 7. Bezahlbare und saubere } \\
\text { Energie }\end{array}$ & $\begin{array}{l}\text { 13. Maßnahmen } \\
\text { zum Klimaschutz }\end{array}$ & \multirow{6}{*}{$\begin{array}{l}\text { 16. Frie- } \\
\text { den, } \\
\text { Gerech- } \\
\text { tigkeit } \\
\text { und } \\
\text { starke } \\
\text { Institu- } \\
\text { tionen }\end{array}$} & \multirow{6}{*}{$\begin{array}{l}\text { 17. Part- } \\
\text { nerschaf- } \\
\text { ten zur } \\
\text { Errei- } \\
\text { chung der } \\
\text { Ziele }\end{array}$} \\
\hline 2. Kein Hunger & $\begin{array}{l}\text { 8. Menschenwürdige } \\
\text { Arbeit und Wirtschafts- } \\
\text { wachstum }\end{array}$ & $\begin{array}{l}\text { 14. Leben unter } \\
\text { Wasser }\end{array}$ & & \\
\hline $\begin{array}{l}\text { 3. Gesundheit und } \\
\text { Wohlergehen }\end{array}$ & $\begin{array}{l}\text { 9. Industrie, Innovation } \\
\text { und Infrastruktur }\end{array}$ & \multirow[t]{4}{*}{$\begin{array}{l}\text { 15. Leben an } \\
\text { Land }\end{array}$} & & \\
\hline $\begin{array}{l}\text { 4. Hochwertige Bil- } \\
\text { dung }\end{array}$ & $\begin{array}{l}\text { 10. Weniger Ungleichhei- } \\
\text { ten }\end{array}$ & & & \\
\hline $\begin{array}{l}\text { 5. Geschlechtergleich- } \\
\text { heit }\end{array}$ & $\begin{array}{l}\text { 11. Nachhaltige Städte } \\
\text { und Gemeinden }\end{array}$ & & & \\
\hline $\begin{array}{l}\text { 6. Sauberes Wasser } \\
\text { und Sanitäreinrichtun- } \\
\text { gen }\end{array}$ & $\begin{array}{l}\text { 12. Nachhaltiger Konsum } \\
\text { und Produktion }\end{array}$ & & & \\
\hline
\end{tabular}

Nichtübereinstimmung wurden die fraglichen Textstellen in einer Kodierkonferenz besprochen (Mayring 2020, S. 499). Dies war in erheblichem Ausmaß notwendig, da sich der Kodierprozess als schwierig erwies. Viele Aspekte aus den Positionspapieren konnten verschiedenen SDGs zugeordnet werden, oder es bedurfte umfassender Auslegung, ob die Aspekte tatsächlich unter die entsprechenden Nachhaltigkeitsindikatoren zu subsumieren sind. Die Auswertung und Dokumentation der Kategorien erfolgten computergestützt mit dem Programm MAXQDA (VERBI Software, Berlin, Deutschland).

Als Auswahl- und Analyseeinheiten (Kuckartz 2018, S. 30) zur Charakterisierung und Definition eines Positionspapiers zur Lausitz wurden folgende Kriterien bestimmt: Erstens müssen die Positionspapiere in schriftlicher Form vorliegen. Mit dem Kriterium der schriftlichen Veröffentlichung wird eine gewisse Kontinuität, Transparenz, Ernsthaftigkeit und die Möglichkeit einer weiten Verbreitung gewährleistet und zum Ausdruck gebracht. Als zweites Kriterium wurde bestimmt, dass die Positionspapiere aus Lausitzer Akteur*innenzusammenhängen entstanden sind, wobei dies auf Grund von mangelnden polnischen Sprachkenntnissen der Wissenschaftler*innen auf Brandenburg und Sachsen beschränkt bleiben musste. Drittens wurden für die Dokumentenanalyse keine wissenschaftlichen Studien oder Gutachten herangezogen, da die große Anzahl der wissenschaftlichen Gutachten das hier beschriebene Vorhaben verzerren würde und die Auffassung der regionalen Akteur*innen im Zentrum des Interesses steht. Viertens erfolgte für die analysierten Dokumente die zeitliche Eingrenzung von 2015 bis August 2020. Diese Zeitspanne markiert den Zeitraum von der Veröffentlichung des Eckpunktepapiers des Bundesministeriums für Wirtschaft und Energie zum Strommarkt aus dem Jahr 2015, das die Energiewende wesentlich mit einleitete, bis zum August 2020 als dem Zeitpunkt, an dem das Kohleausstiegsgesetz und das Strukturstärkungsgesetz Rechtskraft erlangten.

Die Sammlung der Positionspapiere erfolgte durch eine Internetrecherche sowie durch eine gezielte Telefonrecherche bei Akteur*innen, die als wichtig eingeschätzt wurden. Insgesamt konnten auf diese Weise 17 Positionspapiere in die Inhaltsanalyse 
einbezogen werden $(n=17)$. Vier Positionspapiere sind von politischen Parteien verfasst $^{4}$. Vier Positionspapiere entstammen wirtschaftsnahen Interessenverbänden ${ }^{5}$. Vier Positionen konnten zivilgesellschaftlichen Vereinen und Verbänden zugeordnet werden $^{6}$. Drei Papiere entstammen kommunalen Arbeitszusammenhängen ${ }^{7}$ und zwei sonstige Papiere auf Ebene der Bundesländer ${ }^{8}$, die auf den Kohleausstieg Bezug nehmen. Es ist anzunehmen, dass bei diesen Gruppen jeweils unterschiedliche Grade der Betroffenheit vorliegen bzw. verschiedene Ebenen (Kommunen, Land, Bund) eine andere Intensität der Auseinandersetzung mit dem Strukturwandel erzeugen. Auch kann nichts über den Wirkungsgrad oder die Resonanz und Rezeption der einzelnen Papiere ausgesagt werden. Diesen Aspekten konnte in der Auswertung nicht Rechnung getragen werden.

\section{Ergebnisse der qualitativen Inhaltsanalyse}

Im Folgenden wird dargestellt, wie die fünf Kernbotschaften aus den 17 SDGs in den Positionspapieren der Lausitzer Akteur*innengruppen als Codes aufgefunden wurden. Dabei erfolgt zunächst eine Häufigkeitsdarstellung (Tab. 2). Anschließend wird aufgeführt, wie sich die einzelnen Kernbotschaften der SDGs in den Lausitzer Positionspapieren inhaltlich darstellen.

\footnotetext{
${ }^{4}$ Bei den Parteien wurden lediglich speziell auf den Lausitzer Strukturwandel verfasste Papiere auf Landesebene in die Analyse einbezogen: 1) „Initiativgruppe Energiewende der CDU Brandenburg (2015)“, 2) „Perspektiven für die Lausitz nach der Kohle - Grüne Impulse für den Strukturwandel einer Region von den GRÜNEN in Sachsen (2015)“ 3) „Unsere Lausitz für Morgen - für eine aktive Strukturpolitik: Vernetzt, Kooperativ, Innovativ von der SPD in Sachsen (2017)“ und 4) „Für eine liebenswerte Lausitz Vorschläge und Ideen für den Strukturwandel von der Partei Die Linke in Brandenburg (2019)“.

5 Die Positionspapiere aus wirtschaftsbezogenen Interessensverbände sind: 5) „Ideen, Investitionen, Infrastruktur. So gelingt der Strukturwandel in der Lausitz“ der Unternehmensverbände Berlin-Brandenburg, 6) „Zukunftsvision Lausitz: Starkes Handwerk. Perspektivreiche Region“ der Handwerkskammern Cottbus und Dresden, 7) „IG BCE Positionspapier zur WSB-Kommission“ und 8) „Forderungen des Mittelstands an die Kohlekommission" von dem Bundesverband mittelständische Wirtschaft und Unternehmerverband Deutschland e. V. (BVMW 2018).

6 Bei den Vereinen konnten 9) vom Verein Pro Lausitz e.V „Ein Herz für saubere Lausitzer Kohle“, 10) das Papier „Strukturentwicklung in der Lausitz. Empfehlungen für einen Strukturplan im Bereich Sorben/ Wenden“ durch das Sorbische Institut, 11) Die Stellungnahme zum Entwurf eines Strukturstärkungsgesetzes Kohleregionen der Deutschen Umwelthilfe e. V. und 12) die „Lausitzer Erklärung“ des Vereins „Lausitzer Perspektiven“ als Positionspapiere einbezogen werden (Lausitzer Perspektiven o.J., Verein Pro Lausitz 2017).

7 Hier handelt es sich um 13) das Leitbild der Stadt Cottbus, 14) die Hohenmölsener Erklärung zum Braunkohlestrukturwandel durch Bürgermeister der Revierstädte sowie 15) der gemeinsame Apell der Bürgermeister*nnen der drei Braunkohlereviere in der Bundesrepublik Deutschland.

8 Im Bereich der sonstigen Positionspapiere handelt es sich um 16) den Bericht des Lausitz-Beauftragten des Landes Brandenburg sowie um 17) die Stellungnahme der Evangelische Kirche Berlin, Brandenburg, Schlesische Oberlausitz (EKBO 2020) zur Braunkohle.
} 
Tab. 2 Verteilung der Kernbotschaften in den Lausitzer Positionspapieren (eigene Darstellung)

\begin{tabular}{|c|c|c|c|c|c|c|c|c|}
\hline People & & Prosperity & & Planet & & Peace & & Partnership \\
\hline $\begin{array}{l}\text { 1. Keine } \\
\text { Armut }\end{array}$ & 0 & $\begin{array}{l}\text { 7. Bezahlbare } \\
\text { und saubere } \\
\text { Energie }\end{array}$ & 22 & $\begin{array}{l}\text { 13. Maß- } \\
\text { nahmen } \\
\text { zum Kli- } \\
\text { maschutz }\end{array}$ & 8 & \multirow{6}{*}{$\begin{array}{l}16 . \text { Frie- } \\
\text { den, } \\
\text { Gerech- } \\
\text { tigkeit } \\
\text { und } \\
\text { starke } \\
\text { Institu- } \\
\text { tionen }\end{array}$} & 19 & \multirow{6}{*}{$\begin{array}{l}\text { 17. Part- } \\
\text { ner- } \\
\text { schaften } \\
\text { zur Er- } \\
\text { reichung } \\
\text { der Ziele }\end{array}$} \\
\hline $\begin{array}{l}\text { 2. Kein Hun- } \\
\text { ger }\end{array}$ & 0 & $\begin{array}{l}\text { 8. Menschen- } \\
\text { würdige } \\
\text { Arbeit und } \\
\text { Wirtschafts- } \\
\text { wachstum }\end{array}$ & 59 & $\begin{array}{l}\text { 14. Leben } \\
\text { unter } \\
\text { Wasser }\end{array}$ & 0 & & & \\
\hline $\begin{array}{l}\text { 3. Gesund- } \\
\text { heit und } \\
\text { Wohlergehen }\end{array}$ & 11 & $\begin{array}{l}\text { 9. Industrie, } \\
\text { Innovation } \\
\text { und Infra- } \\
\text { struktur }\end{array}$ & 61 & $\begin{array}{l}\text { 15. Leben } \\
\text { an Land }\end{array}$ & 5 & & & \\
\hline $\begin{array}{l}\text { 4. Hochwer- } \\
\text { tige Bildung }\end{array}$ & 40 & $\begin{array}{l}\text { 10. Weniger } \\
\text { Ungleichhei- } \\
\text { ten }\end{array}$ & 12 & & & & & \\
\hline $\begin{array}{l}\text { 5. Ge- } \\
\text { schlech- } \\
\text { tergleichheit }\end{array}$ & 4 & $\begin{array}{l}\text { 11. Nachhal- } \\
\text { tige Städte } \\
\text { und Gemein- } \\
\text { den }\end{array}$ & 29 & & & & & \\
\hline $\begin{array}{l}\text { 6. Sauberes } \\
\text { Wasser und } \\
\text { Sanitärein- } \\
\text { richtungen }\end{array}$ & 2 & $\begin{array}{l}\text { 12. Nachhal- } \\
\text { tiger Konsum } \\
\text { und Produkti- } \\
\text { on }\end{array}$ & 7 & & & & & \\
\hline $\begin{array}{l}\text { Summe der } \\
\text { Codes: } 57\end{array}$ & & $\begin{array}{l}\text { Summe der Cod } \\
190\end{array}$ & & $\begin{array}{l}\text { Summe der } \\
\text { Codes: } 13\end{array}$ & & $\begin{array}{l}\text { Summe der } \\
\text { Codes: } 19\end{array}$ & & $\begin{array}{l}\text { Summe der } \\
\text { Codes: } 25\end{array}$ \\
\hline
\end{tabular}

\subsection{Quantitative Verteilung der 17 SDGs innerhalb der Positionspapiere}

Es wurden 304 Einzelkodierungen in den Positionspapieren vorgenommen. Mit 190 Einzelkodierungen im Bereich Prosperity ist die Kernbotschaft der wirtschaftlichen Entwicklung am häufigsten genannt worden. Dem folgt die Kernbotschaft People mit 57 Kodierungen. Allen anderen Kernbotschaften konnte eine wesentlich geringere Anzahl an Kodierungen zugeordnet werden. Diese Gewichtung lässt sich auf verschiedene Ursachen zurückführen. Zum einen entstammt ein Großteil der Positionspapiere dem Bereich wirtschaftlicher Interessensvertretungen, sodass die Aufmerksamkeit auf den Bereich der Wirtschaft durchaus nachvollziehbar ist. Zum anderen ist der Anlass der Positionspapiere - der Lausitzer Strukturwandel in Folge des Kohleausstiegs - vor allem eine Transformation im industriellen Energiesektor und bedeutet, dass neue Bereiche der wirtschaftlichen Wertschöpfung erschlossen werden müssen. Es ist daher plausibel, dass als Forderungen in den Positionspapieren besonders jene Bereiche vertreten sind, die den SDGs 9 „Industrie, Innovation und Infrastruktur“ (61 Kodierungen), gefolgt von SDG 8 ,Menschenwürdige Arbeit und Wirtschaftswachstum“ (59 Kodierungen) und SDG 4 ,hochwertige Bildung“ (40) entsprechen, die diesen Bereich kompensieren können. Damit im Zusammenhang steht auch die Relevanz der Kernbotschaften Peace und Partnership, denn die quantitative Betrachtung zeigt die Relevanz der interregionalen und vor allem 
osteuropäischen Vernetzung, der nachhaltigen demokratischen Gestaltung von Gemeinwesen sowie der vielfältigen Entwicklungen kulturellen Lebens in den Lausitzer Positionspapieren deutlich auf.

Gleichwohl handelt es sich um eine extrem polare Gewichtung, denn die Aspekte der Kernbotschaft Prosperity sind gegenüber den Aspekten der Kernbotschaft People mehr als dreimal so häufig in den Positionspapieren auffindbar. Die Nennung der Kernbotschaft Prosperity übersteigt die Nennungen in Bezug auf die Kernbotschaft Planet sogar um das Zehnfache. Auch diese Gewichtung kann zum Teil damit erklärt werden, dass nur zwei Positionspapiere (Bündnis 90/die Grünen Sachsen (2015) und die Deutsche Umwelthilfe e. V. 2020) aus einer explizit ökologischen Interessensvertretung stammen. Gleichwohl zeigt sich, dass - zumindest auf der Ebene einer formal quantitativen Ebene der Beschreibung des Diskurses in Form von öffentlich zugänglichen Positionspapieren - die Thematisierung des Strukturwandels in der Lausitz vom Bereich der Wirtschaft sehr deutlich dominiert wird. Es besteht die Gefahr, dass der Bereich Planet - nachdem der Ausstieg aus Tagebau und Kohleverstromung als Ursprung für den Strukturwandel gewertet wird - in der Folge vernachlässigt wird.

\subsection{Qualitative Beschreibung der 17 SDGs innerhalb der Positionspapiere}

Im Folgenden werden nun die inhaltlichen Aspekte, die in den Positionspapieren zu den 17 SDGs genannt wurden, aufgeführt. Die Darstellung beansprucht keine Vollständigkeit in Bezug auf kodierte Aspekte, sondern zielt darauf ab, auf Basis der Inhaltsanalyse eine Beschreibung der in den Positionspapieren postulierten $\mathrm{Zu}$ kunftsperspektiven, die im Rahmen der Kernbotschaften der SDGs verortet werden können, zu vermitteln.

\subsubsection{Die Kernbotschaft People}

Aspekte, die sich unter die SDG 1 „Keine Armut“ und SDG 2 „Kein Hunger“ subsumieren ließen, waren kein Thema in den Positionspapieren zum Lausitzer Strukturwandel. Bezüglich des SDG 3 ,Gesundheit und Wohlergehen“ heißt es im Leitbild der Stadt Cottbus: „Als Standort des größten Klinikums Südbrandenburgs nutzt Cottbus die mit den Einrichtungen für Forschung und Lehre sowie die mit dem benachbartem Naturraum gegebenen Chancen für die Entwicklung der Gesundheitswirtschaft (2017, S. 18)“. Die Partei Die Linke in Brandenburg sieht ,eine gute und flächendeckende gesundheitliche Versorgung“ (2019, S. 4) als Teil der Daseinsvorsorge und Voraussetzung zur Schaffung zukunftsfähiger und innovativer Arbeitsplätze in der Region. Gemäß des Lausitzbeauftragten des Landes Brandenburg schafft „der vom Land 2015 initiierte Aufbau des Gesundheitscampus Brandenburg [...] neue Chancen für Wissenschaft und Forschung sowie für die gesundheitliche und pflegerische Versorgung in der Region (2018, S. 4). “ Damit rückt die demografische Entwicklung einer älter werdenden Gesellschaft in der Lausitz in den Fokus sowie die Notwendigkeit der Verbindung von Fachkräftesicherung und Innovation im Gesundheitsbereich (Bündnis 90/Die Grünen Sachsen 2015, S. 6). Den Aspekt der gesundheitlichen Daseinsfürsorge bringt die SPD Oberlausitz wie folgt auf den Punkt: 
„Sowohl Alt als auch Jung brauchen einen Arzt in ihrer Nähe“ (2017, S. 6). Auch die Ressourcen der Region als Beitrag zum menschlichen Wohlergehen werden wiederholt thematisiert: „Als überregional interessanter Treffpunkt für Sporttreibende im, auf und am Wasser wird der „Cottbuser Ostsee“9 mit infrastrukturellen, touristischen und wirtschaftlichen Vorhaben gestaltet“" (Leitbild der Stadt Cottbus 2017, S. 23).

SDG 4 „Hochwertige Bildung“ stellte sich als ein sehr relevanter Code in den Lausitzer Positionspapieren heraus. Hierzu schreibt der Unternehmensverbund Berlin-Brandenburg:

„Die Verfügbarkeit von Fachkräften wird in den kommenden Jahren für Unternehmen ein entscheidender Standortfaktor - vor allem in Brandenburg angesichts seiner schwierigen demografischen Entwicklung. Die Herausforderung ist, die Menschen vor Ort zu qualifizieren für neue Anforderungen wie etwa die Digitalisierung. Zudem muss der Fachkräfte-Nachwuchs modern ausgebildet werden. Die Lebens- und Arbeitsbedingungen müssen so gut sein, dass sie talentierte Nachwuchskräfte aus anderen Regionen anziehen. Für neue, wissensbasierte Industriezweige sind Hochschul-Absolventen wichtig. Attraktive Studiengänge, an der BTU und an anderen Einrichtungen, sollen mehr junge Menschen anziehen.“ (2018, S. 4)

Um das Potenzial von Hochschulen und Forschungseinrichtungen für die gesellschaftliche, wirtschaftliche und demographische Entwicklung in der Region zu heben, soll sich im kommenden Jahrzehnt eine Phase des Ausbaus des Hochschulstandorts und des akademischen Bildungsangebots, der Stärkung von Forschung sowie der Etablierung von Transferwissen und -strukturen in der Lausitz anschlieBen. Dabei sollen moderne Studienangebote stärker als bislang Bezüge zur regionalen Wirtschaft beinhalten, gesellschaftliche Bedarfe in der Region befriedigen (z. B. in Bereichen wie Pflege, Betreuung von Kindern und Jugendlichen, Gesundheit) sowie Studienperspektiven für Studierende der Anrainerstaaten aufweisen (Bericht des Lausitzbeauftragten 2018, S. 4). Bedeutsam sei auch der Bereich der beruflichen Bildung. Dazu schreiben Bündnis 90/die Grünen Sachsen: „Die Berufs- und Studienorientierung muss verpflichtend bereits in der Schule beginnen. Nicht leisten kann sich die Lausitz doppelt so viele Schulabgänger ohne Abschluss wie im Bundesschnitt! Jeder einzelne Mensch ist wichtig und muss gefördert werden (Bündnis 90/die Grünen Sachsen 2015, S. 6).“ Es wird von der Partei Die Linke Brandenburg ein dichtes Netz von qualitativ guten Bildungseinrichtungen gefordert, wozu auch Kindertagesstätten mit flexiblen Betreuungszeiten und ein breites Schulangebot von der Grundschule bis zu den Berufsschulen gehören sollen (2019, S. 5). Im gesamten Bildungssystem sei auch die strukturelle „Sicherung und Revitalisierung der niedersorbischen Sprache“ zu berücksichtigen (Sorbisches Institut 2018, S. 6). Die Lausitz soll

,aufbauend auf den Erfahrungen des wissenschaftlich begleiteten Witaj-Projektes in sorbischen Kindertagesstätten das „Modellprojekt 4-Sprachen-Land“

\footnotetext{
${ }_{9}$ Der Cottbuser Ostsee ist mit 1900 ha das größte künstlich entstehende Binnengewässer in Deutschland als Bergbaufolgelandschaft. Dies soll bis 2030 abgeschlossen sein.
} 
begründen. Bereits von der Kita an wird die durchgehende Mehrsprachigkeit über die Grundschule bis in alle weiterführenden Schulen in den Sprachen Deutsch, Sorbisch, Polnisch und/oder Tschechisch garantiert. Um dies zu erreichen, muss es möglich werden, Erzieherinnen und Erzieher aus den Nachbarländern in der Ober- und Niederlausitz einzustellen. Dazu müssen die entsprechenden Berufsabschlüsse in Deutschland endlich anerkannt werden." (Bündnis 90/Die Grünen Sachsen 2015, S. 9).

Themen im Kontext des SDG 5 „Geschlechtergerechtigkeit“ werden relativ gering thematisiert, und wenn, dann vor allem in wirtschaftlicher Perspektive. Gefordert wird: „Insbesondere für junge Frauen müssen Karrierechancen in der Region erlebbar werden“ (der Lausitzbeauftragte 2018, S. 8) und die Partei Bündnis 90/Die Grünen Sachsen möchten ,ein Förderkonzept für unternehmerisches Handeln von Frauen initiieren“ (2015, S. 5). In Bezug auf SDG 6 ,,Sauberes Wasser und Sanitäreinrichtungen“ schreiben Bündnis 90/Die Grünen Sachsen: „Die Verockerung und Versauerung der Spree bedrohen den Tourismus im Spreewald. Deshalb muss neben Sofortmaßnahmen vor Ort die Bekämpfung der Schäden bei den aktuellen Braunkohletagebauen nachhaltig angegangen werden (2015, S. 11)."Es sei - so die Linke Brandenburg - ,noch Jahrzehnte ein funktionierendes Wassermanagement erforderlich. Eine klare Spree von der Mündung bis Berlin, eine durch die Öffentlichkeit zu nutzende Seenkette sowie ein sich selbst regulierender Wasserhaushalt sind hierfür die Voraussetzungen“, um dem „Absenken des Grundwasserspiegels durch die Braunkohlenutzung oder der Sulfat- und Eisenockerproblematik“ entgegenzuwirken (2019, S. 7).

Zusammengenommen geht es im Bereich People in erster Linie darum, die sozialen Rahmenbedingungen für wirtschaftliches Handeln sicherzustellen. Dabei hebt sich zum einen der Bereich der Gesundheit und zum andern der Bereich der Bildung als wichtige Größe hervor. Als „People“ werden über die Absicherung der gesundheitlichen Daseinsfürsorge im Kontext des demographischen Wandels vor allem ältere Menschen, im Sinne eines attraktiven Arbeitsortes die Mitarbeiter*innen der Gesundheitswirtschaft und im Sinne eines Wohnortes mit guter gesundheits- und bildungsbezogener Infrastruktur mögliche Zuzügler*innen in die Lausitz adressiert. Über den Bereich der Bildung werden für junge Menschen vorschulische, schulische, hochschulische und ausbildungsbezogene Entwicklungsbedarfe thematisiert.

\subsubsection{Die Kernbotschaft Prosperity}

Dass die Lausitz Energieregion bleiben und von der Energiewende profitieren kann und soll, ist die gemeinsam geteilte Auffassung in den Positionspapieren hinsichtlich SDG 7 „Bezahlbare und saubere Energie“. So heißt es im Leitbild der Stadt Cottbus: „Regenerative Energieträger und innovative Energiedienstleistungen werden zu weiteren wichtigen Standortfaktoren für Cottbus und die Region entwickelt (2017, S. 18)“. Dabei sollen nur Planungsverfahren für Gewerbe- und Energieerzeugungsstandorte zulässig sein, die der Erforschung, Nutzung und Entwicklung erneuerbarer Energien dienen (Bürgermeister der drei Reviere 2020, o. S.). Der Lausitzbeauftragte des Landes Brandenburg benennt in diesem Zusammenhang explizit 
„Netzinfrastrukturen, inkl. Systemführung und -management“, „Wasserstoff/grünes Gas“ und „Power-to-X-Anwendungen“ (2018, S. 6). Die IG Bergbau Chemie Energie (IG BCE) hebt hervor, dass die Energiewirtschaft „nur für ein Drittel des $\mathrm{CO}_{2-}$ Ausstoßes verantwortlich“ sei (2018, o. S.), und im Positionspapier der Mittelstandsvereinigung (BVMW) wird die Platzierung großer erneuerbarer Energienanlagen in der Nähe der stillgelegten Kohlekraftwerke gefordert, damit dort die bestehende Netzinfrastruktur genutzt werden kann (2018, o. S.). Für das SDG 8 „Menschenwürdige Arbeit und Wirtschaftswachstum" waren die folgenden Aspekte innerhalb der Lausitzer Positionspapiere bedeutsam. Im Positionspapier der SPD Oberlausitz heißt es, das zentrale Ziel sei es, die Wertschöpfung in der Region zu erhalten und auszubauen (2017, S. 2). Der Fokus auf die Sicherung menschenwürdiger Arbeit ist insbesondere auch ein Argument für den Zuzug (hoch)qualifizierter Fachkräfte von außen. So heißt es bei dem Brandenburger Lausitzbeauftragten, dass ,die Städte sowie ländlichen Räume in der Lausitz eng verflochten zu einer „Modellregion der Zukunft" entwickelt werden [sollen] (2018, S. 9). Bezüglich der in der Braunkohle tätigen Personen findet sich die folgende Forderung (Hohenmölsener Erklärung der Bürgermeister der Revierstädte zum Braunkohlestrukturwandel [Hohenlöser Erklärung 2020]): „Die Vereinbarung über ein APG (Anpassungsgeld) für die Direktbeschäftigten in der Braunkohle begrüßen wir ausdrücklich und freuen uns gemeinsam mit den Gewerkschaften über eine dauerhafte Absicherung der Beschäftigten, die in den kommenden Jahren ihre Jobs verlieren werden (2020, o. S.) “. Im gemeinsamen Positionspapier der Handwerkskammern Cottbus und Dresden wird die Förderung von „Regionale[n] Wertschöpfungsketten mit einem ausgewogenen Branchenmix aus Industrie, Dienstleistungen und Handwerk“ gefordert mit dem Ziel, ,die Leistungsfähigkeit des Mittelstands“ (2016, S. 1) zu fördern. Das Sorbische Institut richtet den Blick auf den Bereich der „Kreativwirtschaft und Digitalisierung“ und fordert, dass ,,in die kulturellen Ressourcen investiert werden muss“, da der „Kultur- und Kreativwirtschaft eine wachsende wirtschaftliche Bedeutung" nicht zuletzt auch für den Tourismus zukomme (2018, S. 5). Die Partei die Linke Brandenburg verweist auf die landwirtschaftliche Prägung der Lausitz: „Eine gut funktionierende, vielfältig strukturierte Agrarwirtschaft sowie eine die Region versorgende Gemüseund Obstproduktion sind auch künftig Quelle der Wertschöpfung" (2019, S. 4).

Das SDG 9 „Industrie, Innovation, Infrastruktur“ findet sich in den Positionspapieren zum einen im Hinblick auf eine verschärfte Digitalisierungsstrategie. Zum anderen geht es bei der Frage der Energiewende darum, die bestehende Netzinfrastruktur optimal zu nutzen und den Ausbau der Netze durch eine dezentrale Energiewende auf das Minimum zu reduzieren (BVMW 2018, o. S.). Vor allem aber wird die Lausitz als Wissenschaftsstandort gesehen. Das Handwerk wirbt für eine bessere Verzahnung von Wirtschaft und Wissenschaft, um die Innovationspotenziale im regionalen Mittelstand und Handwerk zu erweitern (HWK CB und DD 2016, o. S.). Die ,enge Zusammenarbeit von Wissenschaft und Wirtschaft“ sei der „Nukleus der Strategie für den Strukturwandel“" (UVB 2018, S. 2). Schließlich gehe es um die Verkehrsinfrastruktur und eine ,schnelle und leistungsfähige Verbindungen zu Metropolen in allen Himmelsrichtungen - im Westen an Leipzig, im Osten an Breslau/ Wroclaw, im Norden an Berlin, im Süden an Dresden. Das sei die Voraussetzung für die Mobilität von Arbeitskräften und Gütern und damit für eine dauerhafte At- 
traktivität als Industriestandort. Für einen raschen Ausbau brauche es einen SonderBundesverkehrswegeplan, der sinnvolle Projekte beschleunigt umsetzt (UVB 2018, S. 5). Die Partei Die Linke Brandenburg postuliert die enge Zusammenarbeit der Lausitzer Kommunen: „Kooperation ist das Schlüsselwort. Jeder „Kleinstaaterei“ erteilen wir eine Absage, denn wir wollen über Gemeindegrenzen hinweg eine Marke „Lausitz“ entwickeln“ (2019, S. 8).

Mit Bezug auf das SDG 10 ,Weniger Ungleichheiten“ wird in den Positionspapieren von vielen Akteursgruppen die Bedeutung der sorbisch/wendischen Sprache und Kultur hervorgehoben. Von den Sorben-Wenden wird die Vision einer „Europäischen Minderheiten-Kompetenzregion für deutsch-slawische Mehrsprachigkeit““ (2018, S. 8) entwickelt.

Im Leitbild der Stadt Cottbus werden verschiedene Ziele im Sinne des SDG 11 „Nachhaltige Städte und Gemeinden“ aufgeführt: So wird ,ein zukunftsorientierter, sicherer sowie sozial- und umweltverträglicher Verkehr für eine Stadt der kurzen Wege" entwickelt und darauf verwiesen, dass der Park Branitz in die UNESCOWelterbeliste aufgenommen werden sollte (2017, S. 7ff.). Die Deutsche Umwelthilfe möchte die Kohleregionen ,zu Modellregionen für öffentlichen Verkehr, für alternative und erneuerbare Antriebsarten“ machen (2019, o. S.). „Kommunen sowie Bürgerinnen und Bürger müssen unterstützt werden, die Energieerzeugung selbst in die Hand zu nehmen“ (2015, S. 8), schreiben Bündnis 90/die Grünen Sachsen. Dem Lausitzbeauftragten ist die „Schaffung von Unterbringungsmöglichkeiten, insbesondere für Auszubildende und Studierende sowie von attraktivem Wohnraum“ (2018, S. 9) wichtig. Die SPD Oberlausitz möchte „,neue Wege für Kultur im ländlichen Raum beschreiten“ (2017, S. 5). Hierbei gelte es, dass sich Kommunen im Strukturwandel ,als Lern- und Erfahrungsräume verstehen und damit die Chance eröffnen, lausitzspezifische Lösungen zu entwickeln und auszuprobieren“ (Bündnis 90/die Grünen Sachsen 2015, S. 9).

Das SDG 12 „Nachhaltiger Konsum und Produktion“ wird in den analysierten Positionspapieren vorrangig mit nachhaltiger Landwirtschaft und nachhaltigem Tourismus in Verbindung gebracht. Bündnis 90/Die Grünen Sachsen schreiben:

„Statt Großmastanlagen können durch artgerechte Tierhaltung und ökologische Landwirtschaft neue Arbeitsplätze entstehen. Die Nachfrage danach wächst ständig. Andere Regionen machen es längst vor: Wertschöpfung gelingt durch die umfangreiche Vermarktung regionaler Produkte mit Hilfe gezielter Anschubfinanzierung. [...] In der Vielfalt der Angebote sind der natur- und landschaftsbezogene sanfte Tourismus mit Angeboten wie Urlaub auf dem Lande oder Radtourismus sowie der Bildungstourismus besonders zu entwickeln“ (2015, S. 6).

Die Partei die Linke Brandenburg positioniert sich wie folgt:

„Wir setzen auf einen Dialog zwischen den in der Landwirtschaft Beschäftigten und den Verbrauchern, der klarstellt, welche Landwirtschaft wir künftig wollen. [...] Wir streiten für eine Bodenpolitik, die den ortsansässigen Landwirten eine Bewirtschaftung ihrer Flächen sichert. Wir wollen eine Vielfalt von 
Eigentumsformen am Boden und werden den Junglandwirten den Zugang zum Bodenerwerb erleichtern. Hohe Bodenpreise behindern die regionale Landwirtschaft und führen zu Spekulationen mit Flächen. Das lehnen wir ab. Wir setzen auf biologische Vielfalt und nachhaltiges Wirtschaften. Die neu entstehenden landwirtschaftlichen Flächen in der Bergbaufolgelandschaft sollen den Lausitzer Bauern gehören und von ihnen bewirtschaftet werden“ (2019, S. 4).

Mit Blick auf die wirtschaftsbezogene Kernbotschaft Prosperity zeigt sich insgesamt, dass erneuerbare Energien eine wichtige Rolle spielen, sodass der Energiesektor in gewandelter Gestalt für die Lausitz weiterhin als strukturbildend betrachtet wird. Gleichwohl werden auch KMUs im Handwerk, Dienstleistungen, Agrarwirtschaft, Tourismus und die Kunst- und Kreativwirtschaft sowie die Potenziale der Digitalisierung in diesen Branchen als bedeutsam eingeschätzt. Verbindendes Element sind die Orientierung an einer Intensivierung regionaler Wertschöpfungsketten. Darüber hinaus wird Vernetzung als wichtiger Aspekt der wirtschaftlichen Entwicklung thematisiert. Dies bezieht sich zum einen auf die enge Verzahnung der Lausitzer Hochschullandschaft mit der Wirtschaft sowie der Vernetzung von Lausitzer Kommunen untereinander. Das SDG 11 ,nachhaltige Städte und Gemeinden“ im Bereich der wirtschaftsbezogenen Kernbotschaft zeigt die Relevanz verwaltungsbezogener und kommunalen Veränderungen sowie die Bedeutung von kulturellen und öffentlichen Infrastrukturen im Zusammenhang mit einem nachhaltigen Strukturwandel auf.

\subsubsection{Die Kernbotschaft Planet}

Die drei SDGs bezüglich der Kernbotschaft Planet sind nur geringfügig in den Positionspapieren ausgeprägt. Bezüglich des SDG 13 „Maßnahmen zum Klimaschutz“ findet sich in dem gemeinsamen Positionspapier der Handwerkskammern Cottbus und Dresden:

„Das Handwerk bestärkt die Politik darin, die Lausitz zur Vorzeige-Region bei der Umsetzung der Energiewende weiterzuentwickeln. Durch gezielte Maßnahmen im Bereich der Energie- und Ressourceneffizienz kann das Handwerk dabei einen maßgeblichen Beitrag zur Förderung des Klima- und Umweltschutzes leisten und zugleich neue Wertschöpfungsquellen erschließen.“(2016, o. S.).

In gleicher Richtung positioniert sich der Bundesverband mittelständische Wirtschaft: „Die kleinen und mittleren Unternehmen unterstützen deshalb die Umstellung der Wirtschaft auf einen nachhaltigen Wachstumspfad“" (2018, o. S.). Das SDG 14 „Leben unter Wasser“ konnte mit keinem Code innerhalb der Lausitzer Positionspapiere versehen werden, was angesichts der Auswirkungen des Tagebaus auf den regionalen Wasserhaushalt problematisch erscheint. Beim Lausitzbeauftragten werden in Bezug auf das SDG 15 ,Leben an Land“ die Etablierung von Institutionen hervorgehoben. So sei das Forschungsinstitut für Bergbaufolgelandschaften Finsterwalde e. V. (FIB) dafür zuständig, Anpassungsstrategien für Bergbaufolgelandschaften und andere in ihrer Funktion beeinträchtigte Lebensräume bzw. Ökosysteme“ zu entwerfen und durch die Gründung und Ansiedlung des Deutschen Fachverbandes für Agro- 
forstwirtschaft in Cottbus/Chóśebuz würden zusätzlich Kompetenzen zur Einführung innovativer Landnutzungssysteme entwickelt und vermittelt (2018, S. 6). Bündnis 90/Die Grünen in Sachsen fordern: „Die Inanspruchnahme von Landschaft muss selbstverständlich immer unter bestmöglicher Berücksichtigung von Naturschutzinteressen sowie in transparenten Verfahren gemäß Bundesimmissionschutzgesetz und Umweltverträglichkeitsprüfung sowie nach Abwägung der Interessen aller Beteiligten erfolgen“" (2015, S. 8). Deutlich wird, dass sich explizite Überlegungen zur Ressourcenschonung der Umwelt und der Berücksichtigung nachfolgender Generationen über die Frage der erneuerbaren Energien hinaus in den Positionspapieren kaum finden.

\subsubsection{Die Kernbotschaft Peace}

In den Positionspapieren wird eine Reihe von Aspekten genannt, die dem SDG 16 „Frieden, Gerechtigkeit und starke Institutionen“ zugeordnet werden können. So findet sich im Leitbild der Stadt Cottbus: „Ehrenamt zahlt sich aus. Das ehrenamtliche Engagement in Vereinen und der lebendigen Kulturszene wird stärker wertgeschätzt und die Bedeutung von Sponsoring bei Unternehmen und öffentlicher Hand spürbar anerkannt (2017, S. 20).“ Auch Beteiligung ist ein wichtiger Aspekt. So schreibt der Verein „Lausitzer Perspektiven“:

„Wir rufen deshalb Menschen in allen Teilen der Lausitz dazu auf, unsere Begeisterung für die anstehende Gestaltungsaufgabe zu teilen. Gemeinsam wollen wir darüber nachdenken, was es braucht, um ,gutes Leben in der Lausitz“ zu ermöglichen. Und dann wollen wir gemeinsam darum ringen, mit welchen konkreten Schritten wir die Region weiterbringen können." (Lausitzer Perspektiven e.V. o.J., o.S.)

Auch der Mittelstand BVMW fordert die „Einbeziehung aller Betroffenen“ (2018, o.S.). Damit wird deutlich, dass Fragen von Zivilgesellschaft, Partizipation und Beteiligung durchaus Bedeutung für den Lausitzer Strukturwandel zugemessen wird.

\subsubsection{Die Kernbotschaft Partnership}

Die Kernbotschaft Partnership, das SDG 17, wird in den Lausitzer Positionspapieren auf verschiedenen Ebenen angesprochen. Von der Partei Die Linke Brandenburg wird das Ziel der Vernetzung damit begründet, dass die Lausitz ,eine länderübergreifende Region“ ist, die ,älter [ist] als die aktuell gültigen Grenzen“ (2019, S. 7). Diese Verbundenheit habe sich bis in die heutige Zeit bewahrt und sei die Grundlage für viele nachbarschaftliche gemeinsame Projekte (ebd.). Die Partei Bündnis 90/Die Grünen Sachsen hebt hervor: „Die Lausitz ist nicht nur Grenzregion zu Polen und Tschechien, sondern hat mit ihrer Lage inmitten Europas die Chance, eine wirtschaftliche und kulturelle Brückenfunktion nach Osteuropa einzunehmen. Um das Knowhow über die Grenzen hinaus zu nutzen, ist eine Internationalisierung wichtig“ (2015, S. 4). Für eine weitere internationale Vernetzung der Region spräche gemäß des Lausitzbeauftragten ,,das weltweit einmalige Knowhow der Lausitz für die Rekultivierung von Bergbaufolge-Landschaften“(2018, S. 11). In den Positions- 
papieren wird die Lausitz als eine integrierte Region auf Landes- und Bundesebene und im osteuropäischen Kontext betrachtet, die auch international exemplarischen Charakter für Transformationsprozesse hat.

\section{Diskussion der Ergebnisse: Profil des Lausitzer Strukturwandels im Lichte der 17 SDGs}

Die qualitative Inhaltsanalyse von Positionspapieren zum Lausitzer Strukturwandel ergibt ein differenziertes Bild. Deutlich zeigt sich, dass die dort aufgeführten Zukunftsentwürfe über Formen der technologisch- und innovationsorientierten Wirtschaft im Bereich erneuerbarer Energien sowie der klein- und mittelständischen Unternehmen im Bereich der Digitalisierung, der Kultur- und Kreativwirtschaft sowie dem Tourismus und die Land- und Ernährungswirtschaft zum überwiegenden Teil sehr gut anschlussfähig an das UN-Indikatorensystem der 17 SDGs im Bereich Prosperity sind. Wenn den in den Positionspapieren dargelegten wirtschaftlichen Entwicklungsdimensionen auch eine entsprechende Umsetzung folgt, so ist die Lausitz - gemessen an den Kriterien der SDGs - auf einem guten Weg im Sinne einer wirtschaftlichen Nachhaltigkeit als effizienter Nutzung von regionaler Ressourcen. Des Weiteren kann festgehalten werden, dass die analysierten Forderungen aus den Positionspapieren im Kern auch im Einklang mit Analysen und Empfehlungen von wissenschaftlichen Gutachten zur wirtschaftlichen Strukturentwicklung der Lausitz stehen (Greib et. al. 2019; Nagel und Zundel 2020).

Betrachtet man die in den Positionspapieren dargelegten Vorstellungen zu den Kernbotschaften People und Planet, so wird offenbar, dass diese keine eigenständigen Entwicklungsdimensionen darstellen, sondern dem Bereich der wirtschaftlichen Effizienz funktional untergeordnet werden. Die im Rahmen der Kernbotschaft People in den Positionspapieren genannten Aspekte wie Bildung, Daseinsfürsorge und sportliche sowie kulturelle Freizeitangebote sind vorwiegend als Kontextfaktoren einer für diese wirtschaftliche Entwicklung benötigten Strategie zur Gewinnung und Sicherung von Fachkräften ausgerichtet. Und auch die Inhalte aus den Positionspapieren in Bezug auf die ökologische Kernbotschaft beinhalten eine wirtschaftliche Grundorientierung, aus der nicht hervorgeht, wie das Kriterium der Konsistenz im Sinne der Nutzung von Ökosystemen, ohne diese zu zerstören, umgesetzt werden kann. Somit ist auch dieses Ergebnis anschlussfähig an bereits vorliegende wissenschaftliche Veröffentlichungen zu den Wandlungsprozessen in der Lausitz. Dort wird der Mangel der Berücksichtigung integrativer Konzepte der Nachhaltigkeitsforschung und die Notwendigkeit von partizipativer Governance zur Gestaltung des Strukturwandels (Herberg et al. 2020a; Herberg et al. 2020b) ebenso postuliert wie eine Orientierung an „Indikatoren für das Gute Leben in der Lausitz“ (Laschewski et al. 2020).

Fragt man nach den Ursachen dieses Ungleichgewichts, so stellt sich auf Basis der vorliegenden qualitativen Inhaltsanalyse folgender Aspekt heraus: Es werden zwar die Bereiche des Sozialen, der Bildung und der Natur zum Gegenstand einer Thematisierung gemacht, jedoch finden sich kaum Positionspapiere von Lausitzer Akteur*innen aus diesen gesellschaftlichen Sektoren selbst. Dies ist für den Bereich 
des Bildungs- und Sozialsystems noch deutlicher als problematisch zu konstatieren als für den Bereich von Natur und Umwelt. Ließen sich bezüglich der ökologischen Perspektive Positionspapiere aus dem politischen Bereich (Bündnis 90/Die Grünen Sachsen) und von Interessensvertretungen (Deutsche Umwelthilfe e. V.) auffinden, war in Bezug auf den Bereich des Sozialen zum Beispiel weder von dienstleistungsbezogenen (Ver.di) oder bildungsbezogenen (GEW) Gewerkschaften noch von den sechs deutschen Wohlfahrtsverbänden oder von freien Trägern und Initiativen des Sozialbereichs Positionspapiere auffindbar. Es fehlen also die Stimmen wichtiger Akteur*innen des Sozialsektors. Dies stellt sich als Defizit dar, weil Partizipation ein Schlüsselprozess der über die Sozialgesetzgebung legitimierten wohlfahrtsstaatlichen Organisation sozialer personenbezogener Dienstleistungen ist und Organisationen der Sozialen Arbeit darüber Mitwirkung und Beteiligung sowohl auf der Ebene fallbezogener Leistungserbringung als auch auf der Ebene der Sozialplanung im kommunalen infrastrukturellen Kontext erbringen und absichern (Gedrath und Schröer 2010; Schnurr 2018).

Um tatsächlich einen integrierten Strukturwandel unter Beteiligung aller Interessensgruppen zu verwirklichen, ist es unabdingbar, diese Positionen wie auch die Positionen weiterer wichtiger gesellschaftlicher Akteur*innengruppen im Dienste der Absicherung der Multiperspektivität systematisch in die Debatte und in Entscheidungsprozesse um die Zukunft der Lausitz einzubeziehen und ggf. zur Entwicklung von Positionspapieren aufzufordern. Für die Lausitz mit ihrer peripheren und ländlichen Lage wären hier neben den Akteur*innen des Sozialsektors beispielsweise auch Akteur*innen aus der Agrarwirtschaft (Deutscher Bauernverband und Arbeitsgemeinschaft Bäuerliche Landwirtschaft) zu nennen.

Greift man abschließend das Ergebnis der Inhaltsanalyse auf, welches für den Lausitzer Strukturwandel nach dem SDG 9 ,Industrie, Innovation und Infrastruktur“ das SDG 4 ,Hochwertige Bildung“ und das SDG 8 ,Menschenwürdige Arbeit und Wirtschaftswachstum" als quantitativ besonders bedeutsam markiert, und reflektiert dies vor dem Hintergrund der drei Kriterien des Nachhaltigkeitsmodells, also der Frage nach Effizienz, Konsistenz und Suffizienz, so wäre zu fragen, wie Bildungsprozesse - seien es vorschulische, schulische, berufsbildende, hochschulische oder Formen der Fort- und Weiberbildung - und die Lausitzer Arbeitswelt entsprechend entworfen werden können? Eine in diesem Sinne zu gestaltende nachhaltige Lausitzer Bildungs- und Arbeitswelt müsste primär dabei ansetzen, die mit $\mathrm{C}$. W. Müller (2016) einleitend beschriebenen Verlusterfahrungen und die mit Krisen- und Transformationsprozessen verknüpften Ängste und Befürchtungen der Menschen aufzufangen. In diesem Sinne würde es darum gehen, die angestrebten Bildungswege und Arbeitsplätze im Bereich von Gesundheit, regenerativer Energiewirtschaft, Digitalisierung etc. auf allen Qualifikationsstufen mit den erforderlichen Ressourcen auszustatten. Diese Ressourcen beziehen sich sowohl auf eine solide ökonomische Absicherung und Honorierung von Bildungsinvestitionen als auch die Gewährleistung von entsprechenden Bildungsräumen und Bildungszeiten zur Auseinandersetzung mit den eigenen „Lebens- und Arbeitsgeschichten“ (Müller 2016, S. 60). Darauf aufbauend müsste es darum gehen, die Prinzipien von Effizienz, Konsistenz und Suffizienz nicht nur als Lerninhalte, sondern auch als gelebte Praxis in der Verknüpfung von Bildungs-, Arbeits- und Lebenswelt gemeinsam mit den Kommunen 
und den verschiedenen Akteur*innen und der Zivilgesellschaft vor Ort in vielen kleineren und größeren Transformationsprojekten zu gestalten, in denen dann in der Tat soziale und kulturelle sowie ökologische Dimensionen gleichberechtigt mit wirtschaftlichen Aspekten umzusetzen wären. Bezogen auf die AGENDA 2030 wäre die Lausitz dann in der Tat nicht weit entfernt von einem gelingenden Modell integrierter sozialer, ökologischer und wirtschaftlicher Transformation. Ein expliziter Bezug zu den 17 SGDs findet sich allerdings in keinem der Positionspapiere, sodass zu reflektieren wäre, ob diese nicht deutlicher als Orientierungskriterien für den Lausitzer Strukturwandel hinzugezogen werden sollten. Damit könnte das Vorhaben einer Modellregion für den Kohleausstieg auch international vergleichbar dargestellt werden.

\section{Ausblick: zur Bedeutung der Sozialen Arbeit im Kontext nachhaltiger Transformationen}

Gesellschaftliche Transformationen und Veränderungen der Sozialen Arbeit sind aufs Engste miteinander verknüpft. Insofern Transformationsphasen dadurch gekennzeichnet sind, dass ,die bisherigen Begründungs- und Legitimationsmuster in die Krise geraten“ und ,mit entsprechenden Veränderungsaufforderungen konfrontiert werden“ (Kessl 2013, S. 10) stellt sich die Frage, welchen Paradigmenwechsel die Transformationsprozesse auf Grund der ökologischen Krise für die Soziale Arbeit erforderlich macht und inwiefern sie als Teil einer „sustainability science“ (Opielka 2016, S. 41) verstanden werden kann. Nimmt man zur Diskussion dieser Frage die Programmatik der 17 SDGs als Bezugspunkt, so ist grundsätzlich $\mathrm{zu}$ konstatieren ist, dass der Prozess der Analyse und kritischen Auseinandersetzung in Bezug auf das Verhältnis von Sozialer Arbeit und ökologischer Krise im Allgemeinen sowie bezüglich der 17 SDGs der AGENDA 2030 als internationale Programmatik im Besonderen noch am Anfang steht. Gleichwohl kann eine Orientierung auf die SDGs für die Soziale Arbeit durchaus eine produktive Erweiterung ihres Bezugshorizonts darstellen, um sich der Auseinandersetzung um ökologische Transformation in einem ersten Schritt zu nähern. Wie sich am Lausitzer Strukturwandel beispielhaft zeigte, könnten in dieser Region verschärfter Transformationsprozesse insbesondere durch interdisziplinäre und sektorenübergreifende Konzeptionen sowie entsprechende Formen der Zusammenarbeit im Sinne eines integrierten Nachhaltigkeitsverständnisses effiziente, konsistente und suffiziente Wertschöpfungsprozesse entwickelt werden. Dabei erscheint eine Kombination der Orientierung an universalen Prinzipien der Nachhaltigkeit und ihre dezentrale Realisierung mit großen lokalen Gestaltungsspielräumen überzeugend. Voraussetzung dafür ist jedoch, dass erstens die Kernbotschaft People einen wirklich eigenständigen und gleichwertigen Status bekommt und die verschiedenen Bereiche des Sozial- und Bildungssektors im Lausitzer Transformationsprozess sowohl deutlicher angefragt und einbezogen werden als auch sich eindringlicher - u. a. in Form von Positionspapieren - zu Wort melden. Bislang zeichnet sich die in der kritischen Literatur zu den SDGs zu findende Dominanz des Wirtschaftlichen zu Ungunsten des Sozialen und Ökologischen sowie der mangelnden Lösung von Zielkonflikten auch in der hier vorgelegten In- 
haltsanalyse ab. Eine grundsätzlichere Frage für die Soziale Arbeit ist, wie sich die Umsetzung des Prinzips der Suffizienz als Verringerung des menschlichen Ressourcenverbrauchs im Sinne der 17 SDGs der AGENDA 2030 darstellen könnte. Gegebenenfalls ist dann für professionelle Handlungskontexte eine weitere Paradoxie zu konstatieren. Diese bestünde zum einen in einer Dezentrierung vom Subjekt im Sinne eines Bewusstseins für die Vulnerabilität und die Existenzrechte nichtmenschlicher Lebewesen verbunden mit einer Kritik jener gesellschaftlichen Subjektivierungsprozesse, in denen die Logik der Unterwerfung der Natur und der Ausbeutung ihrer Ressourcen sich vollziehen. Zum anderen würde diese eine Konzentration auf das Subjekt im Dienste der Stärkung all jener individuellen und kollektiven Transformationsprozesse beinhalten, die eine suffiziente Lebenspraxis hervorbringen.

Danksagung Ich danke meinen ehemaligen wissenschaftlichen Mitarbeiter*innen Anja Raschke und Holger Thomas für ihre Unterstützung bei der Recherche der Positionspapiere, der Inhaltsanalyse mittels MAXQDA und vieler gemeinsamer Auswertungsgespräche. Ohne ihre Mitwirkung wäre der vorliegende Beitrag nicht möglich gewesen.

Funding Open Access funding enabled and organized by Projekt DEAL.

Open Access Dieser Artikel wird unter der Creative Commons Namensnennung 4.0 International Lizenz veröffentlicht, welche die Nutzung, Vervielfältigung, Bearbeitung, Verbreitung und Wiedergabe in jeglichem Medium und Format erlaubt, sofern Sie den/die ursprünglichen Autor(en) und die Quelle ordnungsgemäß nennen, einen Link zur Creative Commons Lizenz beifügen und angeben, ob Änderungen vorgenommen wurden.

Die in diesem Artikel enthaltenen Bilder und sonstiges Drittmaterial unterliegen ebenfalls der genannten Creative Commons Lizenz, sofern sich aus der Abbildungslegende nichts anderes ergibt. Sofern das betreffende Material nicht unter der genannten Creative Commons Lizenz steht und die betreffende Handlung nicht nach gesetzlichen Vorschriften erlaubt ist, ist für die oben aufgeführten Weiterverwendungen des Materials die Einwilligung des jeweiligen Rechteinhabers einzuholen.

Weitere Details zur Lizenz entnehmen Sie bitte der Lizenzinformation auf http://creativecommons.org/ licenses/by/4.0/deed.de.

\section{Literatur}

AGJ (2020). How dare you? Die Verantwortung der Kinder- und Jugendhilfe für die Umsetzung ökologischer Kinderrechte Retrieved from. https://www.agj.de/arbeitsfelder/kindheit-kinderrechtefamilienpolitik/news/artikel.html?tx_news_pi $1 \% 5 \mathrm{Baction} \% 5 \mathrm{D}=$ detail\&tx_news_pi $\% 5 \mathrm{Bcontroller}$ $\% 5 \mathrm{D}=$ News\&tx_news_pi $1 \% 5 \mathrm{Bnews} \% 5 \mathrm{D}=7284 \& \mathrm{cHash}=8 \mathrm{db} 588 \mathrm{c} 6 \mathrm{afb} 53 \mathrm{a} 6 \mathrm{f} 64 \mathrm{e} 0489320 \mathrm{~b} 8 \mathrm{~b} 63 \mathrm{f}$. Zugegriffen: 14. Mai 2021.

Alheit, P., Bast-Haider, K., \& Drauschke, P. (2004). Die zögernde Ankunft im Westen. Biographien und Mentalitäten in Ostdeutschland. Frankfurt a.M.: Campus.

Beck, U. (1992). Der Konflikt der zwei Modernen. Vom ökologischen und sozialen Umbau der Risikogesellschaft. In T. Rauschenbach \& H. Gängler (Hrsg.), Soziale Arbeit und Erziehung in der Risikogesellschaft (S. 185-204). Neuwied, Kriftel, Berlin: Luchterhand.

Beisheim, M. (2014). Ein Review-Mechanismus für die Post-2015-Ziele nachhaltiger Entwicklung: Vorschläge zu seiner Ausgestaltung. Berlin: Stiftung Wissenschaft und Politik (SWP).

Bericht des Lausitzbeauftragten (2018). Strukturentwicklung in der Lausitz. Bericht der Landesregierung. Landtag Brandenburg, 6. Wahlperiode, Drucksache 6/10239. https://www.parlamentsdokumentation. brandenburg.de/starweb/LBB/ELVIS/parladoku/w6/drs/ab_10200/10239.pdf. Zugegriffen: 3. Aug. 2021.

Bock, K. (2000). Politische Sozialisation in der Drei-Generationen-Familie. Eine qualitative Studie aus Ostdeutschland. Opladen: Leske + Budrich. 
Böhnisch, L. (2020). Sozialpädagogik der Nachhaltigkeit. Eine Einführung. Weinheim, Basel: Beltz Juventa.

Bruns, A. (2019). Das Anthropozän und die große Transformation - Perspektiven für eine kritische raumwissenschaftliche Governance- und Transformationsforschung. In M. Abassiharofteh, J. Baier, A. Göb, I. Thimm, A. Eberth, F. Knaps, V. Larjosto \& F. Zebner (Hrsg.), Akademie für Raumforschung und Landesplanung. Räumliche Transformation: Prozesse, Konzepte, Forschungsdesigns, Bd. 10. Hannover.

Bundesministerium für wirtschaftliche Zusammenarbeit und Entwicklung (BMZ) Die Agenda 2030 für nachhaltige Entwicklung. https://www.bmz.de/de/agenda-2030. Zugegriffen: 1. Aug. 2021.

Bundesregierung (2021). Nachhaltigkeitspolitik der Bundesregierung. https://www.bundesregierung.de/ breg-de/themen/nachhaltigkeitspolitik/agenda-2030-die-17-ziele. Zugegriffen: 1. Aug. 2021.

Bundesverband mittelständischer Wirtschaft, Unternehmensverband Deutschland (BVMW) (2018). Forderungen des Mittelstands an die Kohlekommission. Kernforderungen des Mittelstands. https:// www.bvmw.de/fileadmin/03-Themen/Energie/Dateien/2018_Positionspapier_Forderungen_an_die_ Energiekommission.pdf. Zugegriffen: 2. Aug. 2021.

Bündnis 90/die Grünen Sachsen (2015). Perspektiven für die Lausitz nach der Kohle. GRÜNE Impulse für den Strukturwandel einer Region. https://www.gruene-sachsen.de/fileadmin/media/dokumente/ Themen/Laendlicher_Raum/Perspektiven-fuer-die-Lausitz_Web.pdf. Zugegriffen: 2. Aug. 2021.

Bürgermeisterkonferenz der Tagebauanrainer und Kraftwerksstandorte der drei Braunkohlereviere Lausitz, Mitteldeutsches Revier und Rheinisches Revier (2020). Gemeinsamer Apell von Christine Herntier und Torsten Pötzsch (Lausitzer Revier), Marcel Schneider (Mitteldeutsches Revier) \& Schasa Solbach, Andreas Heller und Thomas Hissel (Rheinisches Revier). https://lausitzrunde.com/images/ PDF/Appell_der_Brgermeister.pdf. Zugegriffen: 3. Aug. 2021.

Bütow, B., Chasse, K. A., \& Lindner, W. (2014). Das Politische im Sozialen. Historische Linien und aktuelle Herausforderungen der Sozialen Arbeit. Opladen, Berlin, Toronto: Barbara Budrich.

Bütow, B., Chassé, K. A., \& Maurer, S. (Hrsg.). (2006). Soziale Arbeit zwischen Aufbau und Abbau. Transformationsprozesse im Osten Deutschlands und die Kinder- und Jugendhilfe. Wiesbaden: VS.

Deutsche Umwelthilfe (2019). Stellungnahme der Deutschen Umwelthilfe zum Entwurf eines Gesetzes zur Änderung des Erneuer. https:/www.duh.de/fileadmin/user_upload/download/Projektinformation/ Energiewende/190822_DUH_Stellungnahme_zum_Entwurf_eines_Strukturst\%C3\%A4rkungsgeset zes_Kohleregionen_final.pdf. Zugegriffen: 2. Aug. 2021.

Die Linke (2019). Für eine liebenswerte Lausitz. Vorschläge und Ideen für den Strukturwandel

Dusseldorp, M. (2017). Zielkonflikte der Nachhaltigkeit. Zur Methodologie wissenschaftlicher Nachhaltigkeitsbewertungen. Karlsruhe: J.B. Metzler.

Eisenmenger, N., Pichler, M., Krenmyr, N., Noll, D., Plank, B., Schalmann, E., \& Gingrich, S. (2020). The Sustainable Development Goals prioritize economic growth over sustainable resource use: a critical reflection on the SDGs from a socio-ecological perspective. Sustainability Science, 15, 1101-1110.

EKBO (2020). Stellungnahme zur Braunkohle. https://www.ekbo.de/themen/stellungnahmen.html. Zugegriffen: 10. Juni 2020.

Elder, M., \& Olsen, S. H. (2019). The design of environmental priorities in the SDGs. Global Policy, 10(1), 70-82.

Filho, W.L. (Hrsg.). (2019). Aktuelle Ansätze zur Umsetzung der UN-Nachhaltigkeitsziele. Berlin Heidelberg: Springer Spektrum.

Fukuda-Parr, S., \& McNeill, D. (2019). Knowledge and politics in setting and measuring the SDGs: introduction to special issue. Global Policy, 10(1), 5-15.

Gedrath, V., \& Schröer, W. (2010). Die Sozialgesetzgebung und die Soziale Arbeit im 20. Jahrhundert. In W. Thole (Hrsg.), Grundriss Soziale Arbeit (S. 863-882). Wiesbaden: VS.

Greib, M., Wörlen, C., Richter, F., Ötsch, R., Witt, U., \& Troost, A. (2019). Struktur- und industriepolitische Alternativen für die Lausitz. In Rosa-Luxemburg-Stiftung (Hrsg.), Nach der Kohle. Alternativen für einen Strukturwandel in der Lausitz (S. 9-73). Berlin: Rosa-Luxemburg-Stiftung.

Handwerkskammern Cottbus und Dresden (2016). Zukunftsvision Lausitz: Starkes Handwerk. Perspektivreiche Region. Gemeinsames Positionspapier der Vorstände der Handwerkskammern Cottbus und Dresden mit Kriterien zur Stärkung der wirtschaftlichen Leistungsfähigkeit der Lausitzregion. https://www.hwk-dresden.de/Portals/0/PDF/Presse/Positionspapier_Wirtschaftsregion-Lausitz_ StarkesHandwerk-PerspektivreicheRegion.pdf. Zugegriffen: 2. Aug. 2021.

Herberg, J., Gabler, J., Gürtler, K., Haas, T., Staemmler, J., Löw, B. D., \& Luh, V. (2020a). Von der Lausitz lernen. Wie sich Nachhaltigkeitsforschung für Demokratiefragen öffnen kann. GAIA, 29(1), 60-62.

Herberg, J., Kamlage, J.-H., Gabler, J., Goerke, U., Gürtler, K., Haas, T., \& Venghaus, S., Beer, D.L., Luh, V., Knobbe, S., Reinermann, J., Staemmler, J. (2020b). Partizipative Governance und nachhal- 
tiger Strukturwandel. Zwischenstand und Handlungsmöglichkeiten in der Lausitz und im Rheinischen Revier. Potsdam: IASS Potsdam.

Herlyn, E., \& Lévy-Tödther, M. (Hrsg.). (2020). Die Agenda 2013 als Magisches Vieleck der Nachhaltigkeit. Systemische Perspektiven. Wiesbaden: Springer Nature.

Hohenlöser Erklärung (2020). Bürgermeister der Revierstädte zum Braunkohlestrukturwandel. https:// zeitzonline.de/news/politik/das-revier-ist-hier/. Zugegriffen: 6. Juni 2020.

Industriegewerkschaft Berbau, Chemie, Energie (IG BCE) Unser Einsatz für Wachstum, Strukturwandel und Beschäftigung. https://2019.igbce.de/vanity/renderDownloadLink/4174/172202. Zugegriffen: 2. Aug. 2021.

Initiativgruppe der CDU Brandenburg (2015). Energiewende - Strukturwandel zukunftsfähig gestalten. Lausitzer Revier muss „Modellregion des Umstiegs“ werden. https://expydoc.com/doc/9148497/ initiativgruppe-der-cdu-brandenburg---hans. Zugegriffen: 2. Aug. 2021.

Kessl, F. (2013). Soziale Arbeit in der Transformation des Sozialen. Wiesbaden: Springer VS.

Kuckartz, U. (2018). Qualitative Inhaltsanalyse. Weinheim, Basel: Beltz Juventa.

Langford, M. (2016). Lost in transformation? The politics of the sustainable development goals. Ethics \& International Affairs, 30(2), 167-176. https://doi.org/10.1017/S0892679416000058.

Laschewski, L., Häfner, D., Held, B., Schürmann, C., \& Zieschank, R. (2020). Indikatoren für das Gute Leben in der Lausitz. Cottbus: Zukunftswerkstatt Lausitz. Ein Projekt der Wirtschaftsregion Lausitz $\mathrm{GmbH}$.

Lausitzer Perspektiven e.V. (o.J.). Lausitzer Erklärung. https://lausitzer-perspektiven.de/wp-content/ uploads/2020/09/Erkla\%CC\%88rung_deutsch_layoutet_fin.pdf. Zugegriffen: 20. Aug. 2020.

Liedholz, Y. (2021). Berührungspunkte von Sozialer Arbeit und Klimawandel. Perspektiven und Handlungsspielräume. Opladen, Berlin, Toronto: Barbara Budrich.

Lutz, R., Wagner, L., Rehklau, C., \& Ross, F. (2018). Handbuch Internationale Soziale Arbeit. Weinheim: Beltz.

Mayring, P. (2020). Qualitative Inhaltsanalyse. In G. Mey \& K. Mruck (Hrsg.), Designs und Verfahren. Handbuch Qualitative Forschung in der Psychologie, (Bd. 2, S. 495-511). Wiesbaden: Springer.

Müller, C.W. (2016). Die Lausitz will ein Ferienparadies werden. Ein Erzählprojekt hilft dabei. Sozial Extra: Zeitschrift Für soziale Arbeit. https://doi.org/10.1007/s12054-016-0110-4.

Nagel, M., \& Zundel, S. (2020). „Wat den Eenen sin Uhl', is den Annern sin Nachtigall“. Ausgewählte Aspekte der Standortqualität der Lausitz. Cottbus: Brandenburgische Technische Universität CottbusSenftenberg.

Olk, T., \& Gensicke, T. (Hrsg.). (2014). Bürgerschaftliches Engagement in Ostdeutschland. Wiesbaden: Springer.

Opielka, M. (2016). Soziale Nachhaltigkeit aus soziologischer Sicht. Soziologie, 45(1), 33-46.

Pedersen, C.S. (2018). The UN sustainable development goals (SDGs) are a great gift to business! Procedia CIRP, 69, 21-24. https://doi.org/10.1016/j.procir.2018.01.003.

Schmieg, E. (2017). External trade policy and the Sustainable Development Goals: implementing the SDGs will meet justified criticisms of globalisation. doi:urn:nbn

Schneidewind, U. (2018). Die große Transformation. Frankfurt am Main: Fischer.

Schnurr, S. (2018). Partizipation. In I. H.-U. Otto, H. Thiersch, R. Treptow \& H. Ziegler (Hrsg.), Handbuch Soziale Arbeit (S. 1126-1137). München: Ernst Reinhardt.

SDP Sachsen (2017). Unsere Oberlausitz für morgen. Für eine aktive Sturkturpolitik: Vernetzt, Kooperativ, innovativ. http://www.spd-fraktion-sachsen.de/wp-content/uploads/2017-06-16-PositionspapierOberlausitz.pdf. Zugegriffen: 2. Aug. 2021.

Seibert, H., Weyh, A., Jost, O., Sujata, U., Wiethölter, D., \& Carstensen, J. (2018). Die Lausitz. Eine Region im Wandel. Bd. 3. Berlin Brandenburg: IAB Institut für Arbeitsmarkt- und Berufsforschung.

Sorbisches Institut (2018). Strukturentwicklung in der Lausitz/ Lyzyca. Empfehlungen für einen Aktionsplan im Bereich Sorben/ Wenden. https://www.serbski-institut.de/de/dnlarchiv/EndberichtStrukturentwicklung-uyca_V4_final.318.pdf. Zugegriffen: 2. Aug. 2021.

Spitzer, H. (2019). Globale Herausforderungen und internationale Soziale Arbeit. Soziales_kapital, 21, 42-58.

Stadt Cottbus (2017). Für Cottbus ein Leitbild-2035. https://www.cottbus.de/.files/storage/file/467b816add9f-45ec-8997-6cc222e3c63a/Leitbild_StadtCottbus_A4_2017_Februar_final.pdf. Zugegriffen: 3. Aug. 2021.

Stevens, C., \& Kanie, N. (2016). The transformative potential of the Sustainable Development Goals (SDGs). International Enfironnemental Agreements, 16, 393-396. 
Unternehmensverbände Berlin-Brandenburg (UVB) (2018). Ideen, Investitionen, Infrastruktur. So gelingt der Strukturwandel in der Lausitz. https:/www.uvb-online.de/de/system/files/downloads_und_ vorschaubilder/positionspapier_lausitz_0.pdf. Zugegriffen: 2. Aug. 2021.

Verch, J. (2020). Perspektiven zu einem nachhaltigkeitsbildenden Professionsverständnis und einer nachhaltigkeitskulturellen Ethik in der Sozialen Arbeit. In B. Völter, H. Cornel, S. B. Gahleitner \& S. Voß (Hrsg.), Professionsverständnisse in der Sozialen Arbeit (S. 170-185). Weinheim Basel: Beltz Juventa.

Verein Pro Lausitz (2017). Ein Herz für saubere Lausitzer Kohle?! https://waldkleeblatt.de/2017/11/05/ ein-herz-fuer-saubere-lausitzer-kohle/. Zugegriffen: 2. Aug. 2021. 